\title{
Solutions to Monthly Problems 11456 and 11457
}

\author{
David H. Bailey* Jonathan M. Borwein ${ }^{\dagger}$
}

October 10, 2009

The Monthly problem \#11456 [1] asks to evaluate

$$
\alpha=\lim _{n \rightarrow \infty} n \prod_{m=1}^{n}\left(1-\frac{1}{m}+\frac{5}{4 m^{2}}\right) .
$$

Numerical computations, using, say, $n=10^{9}$, yields the numerical value $3.6898333 \ldots$... Using this value as input to the Inverse Symbolic Calculator 2.0 tool (available at http://glooscap.cs.dal.ca:8087, one of the output results is the tantalizingly simple expression

$$
\alpha \stackrel{?}{=} \frac{e^{\pi}+e^{-\pi}}{2 \pi} .
$$

Indeed, this result can be established directly by typing the Maple command

$\mathrm{n} *$ product $\left(1-1 / \mathrm{m}+5 /\left(4 * \mathrm{~m}^{2} 2\right), \mathrm{m}=1 \ldots \mathrm{n}\right)$;

which yields the expression

$$
\frac{n \Gamma(n+1 / 2-i) \Gamma(n+1 / 2+i)}{\Gamma^{2}(n+1) \Gamma(1 / 2-i) \Gamma(1 / 2+i)} .
$$

After typing limit $(\%, \mathrm{n}=$ infinity $)$; this reduces to

$$
\frac{1}{\Gamma(1 / 2-i) \Gamma(1 / 2+i)}
$$

which, after simplify $(\%)$; yields the final result:

$$
\frac{\cosh \pi}{\pi} \text {. }
$$

*Lawrence Berkeley National Laboratory, Berkeley, CA 94720, dhbailey@lbl.gov. Supported in part by the Director, Office of Computational and Technology Research, Division of Mathematical, Information, and Computational Sciences of the U.S. Department of Energy, under contract number DE-AC02-05CH11231.

†School of Mathematical and Physical Sciences, University of Newcastle, Callaghan, NSW 2308, Australia, jonathan.borwein@newcastle.edu.au. Supported in part by the Australian Research Council. 
The Monthly problem \#11457 [2] asks to evaluate

$$
F(a, b)=\int_{a}^{b} \arccos \left(\frac{x}{\sqrt{(a+b) x-a b}}\right) \mathrm{d} x
$$

Here again, computer experimentation (using either Mathematica or Maple) yields a number of specific results:

$$
\begin{array}{ll}
F[0, b]=b \pi / 4 \quad \text { for } b \geq 0, \\
F[1, b]=\frac{(b-1)^{2} \pi}{4(b+1)} \quad \text { for } b \geq 1, \\
F[2, b]=\frac{(b-2)^{2} \pi}{4(b+2)} \quad \text { for } b \geq 2, \\
F[3, b]=\frac{(b-3)^{2} \pi}{4(b+3)} \quad \text { for } b \geq 3,
\end{array}
$$

which quickly suggest the "obvious" answer:

$$
F[a, b] \stackrel{?}{=} \frac{(a-b)^{2} \pi}{4(a+b)}
$$

This result can be established directly by the Maple command

factor $(\operatorname{int}(\arccos (x / \operatorname{sqrt}((a+b) * x-a * b)), x=a . b))$ assuming $a>0, a<b$;

\section{References}

[1] Raymond Mortini, "Problem 11456," American Mathematical Monthly, vol. 116, no. 8 (Oct 2009), pg. 747.

[2] M. L. Glasser, "Problem 11457," American Mathematical Monthly, vol. 116, no. 8 (Oct 2009), pg. 747. 